Bridgelall, R., Rafert, J. B., Tolliver, D., "Performance of Hyperspectral Imaging with Drone Swarms,"

Transportation Research Record: Journal of the Transportation Research Board,

https://doi.org/10.1177/0361198118775876, May 30, 2018.

\title{
PERFORMANCE OF HYPERSPECTRAL IMAGING WITH DRONE SWARMS
}

Raj Bridgelall, Ph.D.

Program Director

SMARTSe ${ }^{\text {SM }}$, Upper Great Plains Transportation Institute

North Dakota State University

P.O. Box 863676, Plano, TX 75086

Phone: (408) 607-3214

Fax: (701) 231-1945

Email: raj.bridgelall@ndsu.edu

James B. Rafert, Ph.D.

Professor (Retired)

Department of Physics

North Dakota State University

23666 Currant Dr, Golden, CO 80401

Phone: (720) 238-0080

Fax: (701) 231-1945

Email: bruce.rafert@gmail.com

Denver D. Tolliver, Ph.D.

Director

Upper Great Plains Transportation Institute

North Dakota State University

Fargo, ND 58108

Phone: (701) 231-7190

Fax: (701) 231-1945

Email: denver.tolliver@ndsu.edu

Word count: $5413+2$ Tables $(250$ each $)+4$ Figures $(250$ each $)=6913$ words $(7500 \max )$ Abstract: 139 words (250 max)

TRR Paper number: 18-00750

Submission Date: 2/17/2018 


\begin{abstract}
The ongoing proliferation and diversification of remote sensing platforms offers greater flexibility to select from a range of hyperspectral imagers as payloads. The emergence of low-cost unmanned aircraft systems (drones) and their launch flexibility presents an opportunity to maximize spectral resolution while scaling both daily spatial coverage and spatial resolution simultaneously by operating synchronized swarms. This article presents a model to compare the performance of hyperspectral-imaging platforms in terms of their spatial coverage and spatial resolution envelope. The authors develop a data acquisition framework and use the model to compare the achievable performance among existing airborne and spaceborne hyperspectral imaging vehicles and drone swarms. The results show that, subject to cost and operational limitations, a platform implemented with drone swarms has the potential to provide greater spatial resolution for the same daily ground coverage compared to existing airborne platforms.
\end{abstract}

Keywords: adaptive resolution, autonomous systems, daily ground coverage, pushbroom, resolution agile, sensor fusion, spatial resolution, surveillance, swarm sensing, unmanned aircraft systems

\title{
INTRODUCTION
}

Hyperspectral imaging has the potential to identify land cover materials, obstacles, and dynamic targets that would otherwise be difficult or impossible to discern with conventional image sensors (1). However, the trade-offs between spatial resolution, spectral resolution, and daily coverage for existing hyperspectral imaging platforms pose practical limitations. Applications such as multimodal transportation infrastructure monitoring places high demands on performance in terms of spectral resolution, spatial resolution, and ground coverage rate during daylight hours (coverage per day) (1). Important emerging applications in transportation include condition monitoring of railroads, pipelines, and roadways (2). Complementary applications such as traffic flow modelling, post-disaster evaluation, and environmental health monitoring place similarly high demands on performance (3).

Insufficient spatial or spectral resolution could result in high false positives that lead to unnecessary expenses from conducting verification missions or field inspections in hostile terrain. Practitioners, therefore, will benefit from a comparative framework for hyperspectral image acquisition that can provide practical guidance for the budgeting, acquisition, and configuration of appropriate equipment and sensor packages.

Each so-called hyper-pixel of a hyperspectral image sensor captures the spectrum from radiated or reflected electromagnetic (EM) energy, which includes invisible and visible light (4). The device optically separates the EM spectrum from a scene so that dozens of physical pixels from an image sensor can sample the dispersed energy into closely spaced wavelength bins (5). The two main methods of capturing a hyperspectral scene during the same sensor integration time are the sweeping optical slit (so-called pushbrooming) and snapshot hyperspectral imaging. The pushbrooming method sweeps a linear array of imaging pixels across the scene such that each row of a two-dimensional (2D) planar image sensor captures the same line of the scene in different wavelength bands (6). In contrast, full-frame hyperspectral image sensors use optical filters to subdivide the available 2D planar pixel array into groups of pixels (hyper-pixels), such that each pixel within a group simultaneously integrates energy from a different wavelength band (7). Both techniques require image stitching to assemble complete hyperspectral scenes after a mission. Various methods of geometric and orthographic corrections for image stitching are available (8-9). Subsequently, given the same 2D sensor and assuming that the scene changes relatively slowly (10), the pushbrooming approach can achieve both higher spatial and spectral resolutions. Therefore, 
this article focuses on characterizing the performance of sweeping optical slit methods across different platforms.

Given a desired spectral resolution, constraints in the design of optical systems for hyperspectral image acquisition force an inherent trade off in their daily ground coverage (square-kilometers per day) and the spatial resolution (square-meters per pixel). Spaceborne platforms typically move faster than airborne platforms and cover a broader swath, but the spatial resolution can be several-fold larger. On the other hand, airborne platforms can schedule flight paths and missions with the flexibility needed to achieve higher spatial resolution, but the daily ground coverage per aircraft is substantially less. Handheld spectrometers are available to conduct field inspections by foot to achieve even higher spatial resolution, but the remoteness and ruggedness of some terrain limit their accessibility. Hence, practitioners are currently limited to selecting an appropriate combination of different platforms to achieve the desired trade-off (Figure 1).

Satellites that carry hyperspectral imager payloads are rare and relatively inaccessible for continuous scanning missions. As of 2017, there were only three such satellites, and researchers must complete an application process several months in advance and allow several weeks for data delivery after its collection (11). The growing availability of cube satellites (CubeSat) promises to reduce the cost of spaceborne hyperspectral image acquisition. CubeSats are miniaturized satellites that various manufacturers design to a set of standard specifications so that a single vehicle can launch several of them at once to reduce deployment cost (12). However, their size and power constraints limit the availability and performance of hyperspectral imagers. Manned aircraft equipped with hyperspectral imagers require nearby airports, and scheduling them can result in data collection lags of days to weeks (13).

The emergence of small UAS (sUAS) or small drones and lightweight, low-power hyperspectral imagers for commercial applications promises greater flexibility and a wider range of mobility and spatial resolution options (14). The steady pace of cost reduction and the relaxation of regulations are creating many new application opportunities. In particular, the authors of this article have been experimenting with concepts in swarming unmanned aircraft system (UAS) configurations to reconstruct hyperspectral scenes in three-dimensions (15). One aspect of that development incorporates rapid hyperspectral image classification to adjust the flight altitude adaptively (16). That is, sensor parameters and flight paths change in response to target tracking so that spatial resolution and signal quality improves only for selected scenes, thereby reducing the data load and processing requirements.

Swarm robotics incorporates sensing, artificial intelligence, and local communications to achieve collective behavior directed toward a common goal (17). As swarming operations evolved from the field of artificial swarm intelligence, its implementation involves many individual rules, cost minimization functions, and many constraints that require significant computing capabilities to mimic social insect behaviors in the natural world.

Communications among swarm members involve a system of constant feedback. To focus this article, the authors do not cover the enabling technologies or the related issues of practical swarm operations such as flight path management, airspace access planning, and aircraft refueling (including recharging) strategies. Rather the authors point to a diverse set of important research by others who are advancing those solutions for more effective drone operations (18-19).

The main contribution of this article is a model to evaluate the performance of sweeping aperture hyperspectral-imaging platforms in terms of their daily spatial coverage and spatial resolution envelope. The authors develop a data acquisition framework and use the model to compare the achievable performance among existing airborne and spaceborne hyperspectral imaging vehicles and drone swarms. 
The organization of this paper is as follows: the next section presents fundamentals of the optical geometry and the image acquisition methods that lead to the model for evaluating the performance of sweeping aperture hyperspectral-imaging platforms. Section 3 develops a framework for gathering and translating the available data for each platform into the model parameters. Section 4 presents results from applying the model to compare the achievable performance among platforms. The final section offers conclusions that could benefit others who utilize the model.

\section{METHODS}

This section develops the model by applying fundamentals of the optical geometry and the methods of image acquisition and scene assembly that requires a sweeping aperture

(Figure 2). To follow the steps in the model development, it is convenient to summarize all of the parameters and their units of measure (Table 1).

A hyperspectral scene consists of points $S_{\theta \phi h}$ where the triplet $\{\theta, \phi, h\}$ are the latitude, longitude, and elevation of the geospatial position on the surface $S$. Each point on the surface radiates or reflects electromagnetic (EM) energy with intensity $R_{\lambda}\left(\mathrm{S}_{\theta \phi h}, t\right)$ where $t$ is the integration sample interval and $\lambda$ is wavelength span of the EM energy spectrum where

$$
\lambda=\sum_{i} \lambda_{i}
$$

and $\lambda_{i}$ is a discrete wavelength band $i$ within the spectral range. Hyperspectral image sensors map the aggregate EM reflectance from a ground sample area (GSA) to each pixel $\boldsymbol{p}_{x, y, i}$ of the image sensor at time window $t[n]$ such that:

$$
p_{x, y, i}[n]=R_{\lambda_{i}}\left(G_{x, y}, t[n]\right)
$$

$G_{x, y}$ is the center of the GSA with dimensions $\phi_{\mathrm{x}}$ and $\theta_{\mathrm{y}}$. Hence, the spatial resolution of the GSA is $\phi_{x} \theta_{y}$ meters.

\section{Optical Geometry}

The sensor captures image frame $n$ at discrete times $t[n]$ with a integration interval of $\Delta_{t}$. Each planar photosite $p_{\mathrm{x}, \mathrm{y}}$ of the image sensor is associated with a radiometric vector

$$
\vec{R}_{\lambda}=R_{\lambda_{1}}, R_{\lambda_{2}}, R_{\lambda_{2}}, \ldots
$$

with each element $R_{\lambda i}$ representing the charge accumulation associated with the EM reflectance integrated across the GSA, within the individual spectral bands $\lambda_{i}$.

The focusing element of the generalized optical system has a focal length of $d_{\mathrm{f}}$ and is positioned at a distance $d_{\mathrm{s}}$ above the image plane. The subject in focus will be a distance of $h_{\mathrm{s}}$ away where, for a relatively thin-lens, the approximation is

$$
h_{\mathrm{s}} \cong \frac{1}{d_{\mathrm{f}}^{-1}-d_{\mathrm{s}}^{-1}} \text {. }
$$

For pixels of dimension $c_{\mathrm{x}} c_{\mathrm{y}}$ where $c_{\mathrm{x}}=c_{\mathrm{y}}$, the GSA $G_{\mathrm{A}}$ will be

$$
G_{\mathrm{A}}=\phi_{\mathrm{x}}^{2}=\left[\frac{c_{\mathrm{x}}}{d_{\mathrm{s}}} h_{\mathrm{s}}\right]^{2}=\left[\frac{c_{\mathrm{x}}}{d_{\mathrm{s}}} \frac{1}{d_{\mathrm{f}}^{-1}-d_{\mathrm{s}}^{-1}}\right]^{2}=\left[\frac{c_{\mathrm{x}}}{d_{\mathrm{s}} / d_{\mathrm{f}}-1}\right]^{2}=p_{\mathrm{a}} \frac{1}{\left(d_{\mathrm{s}} / d_{\mathrm{f}}-1\right)^{2}}
$$

Therefore, the GSA is directly proportional to the pixel area $p_{\mathrm{a}}$ and inversely proportional to the ratio of the image-to-focal plane distance. Hence, the optical area magnification factor $M_{\eta}$ is 


$$
M_{\eta}=\left(d_{\mathrm{s}} / d_{\mathrm{f}}-1\right)^{-2}
$$

This magnification depends on the separation distance $\delta$ between the image plane and the focal plane where

$$
\delta=d_{\mathrm{s}}-d_{\mathrm{f}}
$$

Hence, the magnification factor is

$$
M_{\eta}=\left(d_{\mathrm{f}} / \delta\right)^{2}=\left(\frac{d_{\mathrm{f}}}{d_{\mathrm{s}}-d_{\mathrm{f}}}\right)^{2}
$$

The image plane distance constrains at least one dimension in the overall size of the sensor system. The size also depends on the volume required to accommodate optical path folding elements along the focal length. Given an optically symmetric system and square pixels the area magnification factor must be

$$
M_{\eta}=\left(\frac{\phi_{\mathrm{x}}}{c_{\mathrm{x}}}\right)^{2}
$$

Hence, given a swath width of $G_{\mathrm{x}}$, the sensor length $S_{\mathrm{x}}$ must be

$$
S_{\mathrm{x}}=G_{\mathrm{x}} M_{\eta}^{-1 / 2}
$$

The number of pixels per row of the sensor $N_{\mathrm{x}}$ is

$$
N_{\mathrm{x}}=\frac{S_{\mathrm{x}}}{c_{\mathrm{x}}}
$$

Equating Equations (6) and (9) and solving for the image plane distance gives

$$
d_{\mathrm{s}}=d_{\mathrm{f}}\left(\frac{c_{\mathrm{x}}}{\phi_{\mathrm{x}}}+1\right)
$$

\section{Image Acquisition}

For a desired image overlap of $\gamma$ the sample interval $L_{\mathrm{y}}$ along the ground track must be

$$
L_{\mathrm{y}}=\theta_{\mathrm{y}}(1-\gamma)
$$

The length of the ground (nadir) path $L_{\mathrm{G}}$ is a function of the aircraft ground speed $V_{\mathrm{G}}$ and flying time of $T_{\mathrm{f}}$ seconds such that

$$
L_{\mathrm{G}}=V_{\mathrm{G}} T_{\mathrm{f}}
$$

in units of $\mathrm{m} \mathrm{s}^{-1}$. Hence, the number of frames $N_{\mathrm{F}}$ needed along the flight path is

$$
N_{\mathrm{F}}=\frac{L_{\mathrm{G}}}{L_{\mathrm{y}}}+1
$$

Note that there will be two frames if the flight path length is identical to the ground sample interval such that $L_{\mathrm{G}}=L_{\mathrm{y}}$. Given the maximum frame rate $F_{\mathrm{r}}$ and counting the initial frame at the starting position, this is equivalent to

$$
N_{\mathrm{F}}=F_{\mathrm{r}} T_{\mathrm{f}}+1
$$


Combining Equations (13), (15), and (16) and solving for the minimum required frame rate $F_{\text {rm }}$ yields

$$
F_{\mathrm{rm}}=\frac{V_{\mathrm{G}}}{c_{\mathrm{y}} \sqrt{M_{\eta}}(1-\gamma)}
$$

The aperture of a pushbroom type of imaging system typically limits the field-of-view (FOV) to one row of the image sensor or to a linear image sensor array such that the swath height $G_{\mathrm{y}}$ is

$$
G_{\mathrm{y}}=\theta_{\mathrm{y}}=c_{\mathrm{y}} M_{\eta}^{1 / 2} \text {. }
$$

Hence, a single sensor payload would capture images that cover a ground area $W_{\mathrm{a}}$ (squaremeters) of

$$
W_{\mathrm{a}}=N_{\mathrm{x}} G_{\mathrm{x}}\left(L_{\mathrm{G}}+G_{\mathrm{y}}\right) \text {. }
$$

This model is extensible to full-frame image sensor types that utilize a larger sweeping aperture for scene assembly.

Reflecting the ground sampling distances to the image sensor pixel sizes yields

$$
W_{\mathrm{a}}=N_{\mathrm{x}} c_{\mathrm{x}} M_{\eta}^{1 / 2}\left(L_{\mathrm{G}}+c_{\mathrm{y}} M_{\eta}^{1 / 2}\right)
$$

Given the ground speed and flying time of a single platform, and substituting Equation (14) into Equation (20), the expression becomes

$$
W_{\mathrm{a}}=N_{\mathrm{x}} c_{\mathrm{x}}^{2} M_{\eta}\left[1+\frac{V_{\mathrm{G}} T_{\mathrm{f}}}{c_{\mathrm{x}} M_{\eta}^{1 / 2}}\right] .
$$

If the minimum frame rate is achievable, then this expression provides the ground coverage area as a function of the sensor, optical, and flight parameters.

These expressions describe the fundamental trade-offs in sensor pixel area, pixel density, optical system package size, the image capture rate and overlap percentage, the flight time, and the equivalent ground speed of the platform. For the same swath width, the flight altitude of a single platform is directly proportional to the ratio of the image plane distance from the focusing element and the image sensor size such that

$$
h_{\mathrm{s}}=G_{\mathrm{x}} \frac{d_{\mathrm{s}}}{S_{\mathrm{x}}}
$$

Hence, by decreasing the size of the optical system, the image plane distance will decrease. The focal length must also decrease to maintain the same magnification factor. Consequently, the aircraft must fly at a lower altitude to maintain the swath width and GSD. Given the instantaneous field of view (IFOV) denoted $I_{\mathrm{V}}$, the GSD is

$$
\phi_{\mathrm{x}}=2 h_{\mathrm{s}} \tan \left(\frac{I_{\mathrm{V}}}{2}\right)
$$

where $h_{\mathrm{s}}$ is the flight altitude. For small angles, $\tan (\theta) \approx \theta$, hence $\phi_{\mathrm{x}} \approx h_{\mathrm{s}} \times I_{\mathrm{V}}$. The frame rate $F_{\mathrm{r}}$ of the optical system is in frames per second and the maximum speed of the platform is in $\mathrm{m} \mathrm{s}^{-1}$. Hence, the daily ground coverage $W_{\mathrm{s}}$ for swarms containing $A_{\mathrm{n}}$ aircrafts with $\gamma_{\mathrm{s}}$ percentage lateral overlap is 
$W_{\mathrm{s}}=W_{\mathrm{a}} A_{\mathrm{n}}\left(1-\gamma_{\mathrm{s}}\right)$.

This model guides the design of a lateral swarming configuration with overlapping fields-ofview, using identical aircrafts and cameras. Given the scarcity of publicly available data, the simplicity and linear scalability of this model can guide design and budgeting decisions prior to the acquisition of multiple expensive aircrafts and sensor packages to collect hyperspectral data, and to validate performance expectations for specific application objectives.

\section{DATA}

The data available for each type of hyperspectral imaging platform that currently exist is scattered throughout the literature. A review process compiled, calculated, and validated the values of the relevant parameters (Table 2). The next section describes the dataset and explains the derivations of the needed model parameters.

\section{EO-1 Satellite}

The Hyperion imager aboard the Earth Observer-1 (EO-1) satellite provides 220 spectral channels that range from 400 to 2500 nanometers (20). The typical revisit for an area is 16 days. The optical system splits the incoming electromagnetic energy into two beams, each focusing on 256 spatial pixels with 60 -micrometer sides. The GSD is nominally 30 meters; therefore, the IFOV is 42.55 micro-radians (Equation 23). The system records images at the rate of 220 frames per second. At an orbit of $705 \mathrm{~km}$, the effective ground path velocity is $23,760 \mathrm{~km} \mathrm{~h}^{-1}$. At the maximum capture rate of the imaging system of $223.4 \mathrm{~Hz}$, the image overlap will be $1.5 \%$ (21).

Each data collection event (DCE) produces five files that include calibration and image data. The calibration files are pre-image dark collect, post-image dark collect, internal calibration lamp collect, and post-lamp dark collect (22). The timing of a Hyperion DCE before and after the image capture event is summarized in minutes and seconds (mm:ss) format (Figure 3). The total overhead time is 14 minutes and 31 sections. The maximum image capture time is 24 seconds. Therefore, the daily coverage efficiency, which is the ratio of the image capture time to the total camera operation time, is only $2.6 \%$.

Approximately 10 minutes of standby time is required to temperature stabilize the analogue signal processors for signal-to-noise ratio calibration. Spacecraft maneuvering is necessary to orient the optical system for the solar calibration. The maneuvering, calibration, standby, and temperature stabilization constraint of every DCE limit the time efficiency of the image acquisition to $2.7 \%$. The EO-1 can schedule a maximum of 11 DCE per day (22). Hence, the system has an ability to image 14,600 square-kilometers per day. However, the system has the potential to provide up to 96 DCE per 24-hour period with a sun synchronous orbit. At the maximum acquisition rate, the platform could capture up to 114,000 squarekilometers of imagery per 24-hour period.

\section{Manned Aircraft Systems}

The Airborne Visible/Infrared Imaging Spectrometer (AVIRIS) sensor is one of the most popular. It provides 224 spectral channels that range from 360 to 2500 nanometers (23). The flight altitude and the optical geometry of the sensor dictate whether the frame rate or the aircraft speed becomes the bottleneck in daily ground coverage. Operators determine the flight altitude based on barometric conditions, aircraft capabilities, cloud cover, sun angle, and other factors such as local regulations and government policies.

The AVIRIS optical system splits the incoming light into four paths that focus the light onto four individual linear image sensor arrays. The nominal dimensions of the chargecoupled detector $(C C D)$ elements are 200 micron $\times 200$ micron (24). Three 64-element and 
one 32-element detector provide the 224 spectral channels. The device utilizes rotating elements in the optical path to scan a field of view across the ground trajectory while focusing the image on one spatial pixel at a time (25). The scanning operation provides an equivalent of 677 pixels of lateral spatial resolution. The IFOV of the optical system is 1 milliradian. Therefore, when flown at a nominal altitude of $h_{\mathrm{s}}=17$ kilometers on the NASA ER-2 aircraft, the GSD is 17 meters (Equation 23). The ER-2 flies at a nominal ground speed of $730 \mathrm{~km} / \mathrm{h}$ to accommodate a line scan rate of $12 \mathrm{~Hz}$. From Equation (17), the maximum aircraft speed provides less than $1 \%$ of image overlap.

The on-board tape memory capacity of the AVIRIS sensor limits the amount of image capture and recording time to approximately 1.1 hours. Given the maximum flight duration of 6.5 hours, the calculated AVIRIS image capture efficiency is approximately $17 \%$. Therefore, the AVIRIS system is capable of producing a maximum of 9,292 square-km of hyperspectral scene per day. When flown on a Twin-Otter (TO) aircraft at an altitude of $4 \mathrm{~km}$, the AVIRIS will provide a much higher spatial resolution of 4 meters but the daily ground coverage will be reduced to 387 square-kilometers, given the same set of optical system constraints.

\section{Small Unmanned Aircraft Systems}

From Equation (17), the optical system configuration, maximum frame rate, and maximum flight altitude will constrain the maximum ground speed to

$$
V_{\mathrm{G}}=F_{\mathrm{rm}} c_{\mathrm{y}} \sqrt{M_{\eta}}(1-\gamma) \text {. }
$$

The maximum frame rate of a compact hyperspectral imager that is suitable for deployment on sUAS is 250 frames per second (26). Given the aperture $(f / 2.5)$ and slit length $(10.5 \mathrm{~mm})$, the effective focal length is 26 millimeter. The image sensor pixel sizes of 6.5 micrometers and the focal length defines the IFOV as 0.3 milliradian (mr). At the maximum flight altitude of 121.2 meters, the GSD is 3 centimeters (Equation 23). Hence, the ground swath is 48 meters. With a desired frame-overlap of $30 \%$ to enable frame stitching, this optical geometry and the maximum frame rate of the imager limits the ground speed to $5.3 \mathrm{~m} \mathrm{~s}^{-1}\left(19 \mathrm{~km} \mathrm{~h}^{-1}\right)$.

The daily coverage efficiency of a UAS that operators can launch from a mobile platform at the target area will be higher than that for one that relies on the availability of an airport or an airstrip nearby. The additional time overhead includes lifting the payload, reaching a stable velocity at the start of image acquisition, time to descend, and the duration lost to battery replacement. A small rotary aircraft has the greatest agility and could ascend and descend without traveling significant distances horizontally to reach the beginning of their image acquisition coordinates. A suitable rotary aircraft carrying a payload of one kilogram (2.2 lbs.) and using a 22.2-volt battery with 16600 milliamp-hour (mA-h) charge capacity will provide a maximum flight time of approximately 35 minutes (27). The selected reference provides a conservative estimate because battery life will likely improve over time. With an average lift speed of $5 \mathrm{~m} \mathrm{~s}^{-1}$, the rotary craft will reach the maximum allowable flight altitude in 24 seconds. Experienced operators can change batteries in less than one minute. If a launch vehicle can follow the ground path to accommodate rapid descent and battery changes, then the daily coverage efficiency for the rotary craft of this capability will be $94.8 \%$. Even further improvements are achievable if the launch vehicle carries pre-charged vehicles to alternate missions.

The frame rate of the image sensor and the optical geometry limits the maximum ground speed for this platform to $19 \mathrm{~km} \mathrm{~h}^{-1}$. Therefore, the sUAS will image approximately 0.5 square-kilometers per mission. The sun will be at a suitable range of angles to provide proper irradiance for approximately four hours (4). Therefore, operators can fly approximately seven data collection missions per aircraft. Consequently, with the sensor, flight altitude, and battery life constraints, the sUAS platform can provide 3.5 square- 
kilometers of hyperspectral images per aircraft per day. If placed on a larger UAS that can fly at the same altitude as the Twin-Otter, the image sensing system will provide a spatial resolution of one meter and a daily ground coverage of 2,417 square-kilometers.

\section{RESULTS AND DISCUSSION}

Applying the derived parameters from the dataset to the model for each platform produces their daily coverage shown in the last column of Table 1 . The altitude $\left(h_{\mathrm{s}}\right)$ is in kilometers $(\mathrm{km})$, the pixel dimension in micrometers $(\mu \mathrm{m})$, the GSD in meters, the IFOV in milliradians $(\mathrm{mr})$, the frame rate is in frames per second, the ground velocity in $\mathrm{km} \mathrm{h}^{-1}$, the image capture efficiency $\eta_{\mathrm{I}}$, and the daily ground coverage $W_{\mathrm{a}}$ is in square-kilometers. The daily ground coverage and the spatial resolution achievable by each platform is summarized graphically (Figure 4). The figure shows that the smaller IFOV typical of lightweight imaging systems has the potential for providing greater spatial resolution for the same daily ground coverage of existing airborne platforms. The arrow shown indicates the scaling direction of the coverage-resolution bound per Equation (24), for each additional sUAS added to the swarm. The figure also includes the daily ground coverage and spatial resolution of a panchromatic imaging platform aboard the GeoEye-1 satellite for comparison. Hyperspectral imagers force a lower coverage-resolution bound because, given an image sensor, they trade off spatial resolution for spectral resolution. The time overhead needed to begin the actual image acquisition during available natural lighting conditions tends to limit substantially the daily ground coverage of existing aerospace platforms. Hence, system designers must carefully consider their operational details and logistics to minimize the time overhead between data collection events.

Drone swarms can have a distinct advantage if they incorporate mobile launch and refueling/recharging platforms at the deployment site to minimize the time overhead. Positioning the platform at the deployment site before the time of optimal sun angle will minimize the overhead to begin a data capture mission. Furthermore, the launch vehicle could potentially carry two drone fleets to accommodate launches during the time that the previous fleet is landing. This approach will minimize the time between data capture events and increase the daily coverage efficiency per launch system. These considerations offer important insights towards the design optimization of swarming aircraft systems for highefficiency operations. Given their advantages, practitioners must also consider the precautions of using drones. Considerations include compliance with federal and state regulations to assure airspace and public safety. According to the Federal Aviation Administration (FAA) website at the time of this publication, operators must register drones that weigh between 0.55 and 55 pounds, and are not flying under the special rule for model aircrafts. The authors recommend that potential practitioners of the method do regularly visit the FAA website because the situation and rules evolve steadily.

\section{CONCLUSIONS}

Many applications are emerging that demand simultaneously vast ground coverage, high spatial resolution, high spectral resolution, and high data collection efficiencies during available lighting conditions. The continuous cost and size reduction of high-performance electronic and optical systems will lead to a proliferation and diversification of aerospace platforms that afford greater flexibility in trading off performance bounds. Nevertheless, a fundamental trade-off between spatial resolution and daily coverage for hyperspectral imaging acquisition sets a practical limitation that constrains their use. Therefore, using the framework for data acquisition and the performance evaluation model developed in this article can help other researchers and practitioners in their work. 
The finding that drone swarms has the potential to provide greater spatial resolution for the same daily ground coverage of existing airborne hyperspectral imaging platforms points to their potential for use in new applications that demand high-performance imaging. Given an image sensor of some spectral resolution, the flight height, fight speed, and image capture efficiencies fundamentally constrains spatial coverage and spatial resolution. Therefore, an ability to scale economically the swarm size will push the daily spatial coverage dimension without diminishing the spatial or spectral resolutions. Furthermore, the potential for flight path adaptation based on target identification can further improve performance in terms of reducing false positives. However, this potential comes with the caveat that practical and high-performance methods must be in place to stitch hyperspectral image frames into hyperspectral scenes, and to enable the refueling and alternating of swarm fleets, without the need for manual interventions. These are all important and ongoing areas of research that this article does not address.

Future research will use the models to design a variety of swarming configurations to enable the three-dimensional reconstruction of hyperspectral scenes for various applications in transportation.

\section{ACKNOWLEDGEMENTS}

A grant from the United States Department of Transportation supported this research under Grant DTRT13-G-UTC38.

\section{AUTHOR CONTRIBUTION STATEMENT}

The authors confirm contribution to the paper as follows: study conception and design: Raj Bridgelall; data collection: Raj Bridgelall; analysis and interpretation of results: Raj Bridgelall, James Rafert, Denver Tolliver; draft manuscript preparation: Raj Bridgelall. All authors reviewed the results and approved the final version of the manuscript.

\section{REFERENCES}

1. Bridgelall, R., J. B. Rafert, and D. D. Tolliver. Remote Sensing of Multimodal Transportation Systems. Fargo, ND: Mountain-Plains Consortium, North Dakota State University - Upper Great Plains Transportation Institute, 2016.

2. Bridgelall, R., B. J. Rafert, and D. D. Tolliver. Hyperspectral Applications in the Global Transportation Infrastructure. In Proc. European Association for Signal Processing (EUSIPCO), Nice, France, 2015.

3. Bridgelall, R., J. B. Rafert, D. D. Tolliver, and E. Lee. Resolution agile remote sensing for detection of hazardous material spills. Transportation Research Record: Journal of the Transportation Research Board, Vol. 2547, 2016, pp. 49-56.

4. Goetz, F. Three decades of hyperspectral remote sensing of the earth: A personal view. Remote Sensing of Environment, Vol. 113, 2009, pp. S5-S16.

5. Hagen N., and M. W. Kudenov. Review of snapshot spectral imaging technologies. Optical Engineering, Vol. 52, No. 9, 2013, pp. 090901-090901.

6. Barbieux, K., D. Constantin, and B. Merminod. Correction of airborne pushbroom images orientation using bundle adjustment of frame images. International Archives of the Photogrammetry, Remote Sensing \& Spatial Information Sciences, Vol. 41, No. B3, 2016, pp. 813-818.

7. Bareth, G., H. Aasen, J. Bendig, M. L. Gnyp, A. Bolten, A. Jung, R. Michels, and J. Soukkamäki. Spectral comparison of low-weight and UAV-based hyperspectral frame cameras with portable spectroradiometer measurements. In Proceedings of the Workshop on UAV-based Remote Sensing Methods for Monitoring Vegetation, Cologne, Germany, 2015. 
8. Jinmeng, H., and S. Zhong. Research on Image Stitching Algorithm for UAV Ground Station Terminal. In Asian Simulation Conference, Singapore, 2016.

9. Stark, F., M. Rosenberger, P.-G. Dittrich, R. Celestre, M. Hänsel, and G. Notni. Geometrical and spectral data stitching for combining hyperspectral imaging systems. atAutomatisierungstechnik, Vol. 65, No. 6, 2017, pp. 381-395.

10. Lippitt, D., and D. A. Stow. Remote Sensing Theory and Time-Sensitive Information. In Time-Sensitive Remote Sensing, C. D. Lippitt, D. A. Stow and L. Coulter, Eds., New York: Springer, 2015, pp. 1-10.

11. UCS. Satellite Database. Cambridge, MA: Union of Concerned Scientists (UCS), 2017.

12. CubeSat Design Specification, Rev 13, San Luis Obispo CA: California Polytechnic State University, 2015.

13. Kramer, H. J. Observation of the earth and its environment: survey of missions and sensors, New York: Springer, 2002.

14. Colomina, I., and P. Molina. Unmanned Aerial Systems for photogrammetry and remote sensing: A review. ISPRS Journal of Photogrammetry and Remote Sensing, Vol. 92, 2014, pp. 79-97.

15. Bridgelall, R., J. B. Rafert, and D. D. Tolliver. Hyperspectral range imaging for transportation systems evaluation. In SPIE Smart Structures and Materials: Nondestructive Evaluation and Health Monitoring, Las Vegas, NV, 2016.

16. Bridgelall, R., J. B. Rafert, D. D. Tolliver, and E. Lee. Rapid hyperspectral image classification to enable autonomous search systems. Journal of Spectral Imaging, Vol. 5, No. 1, 2016, pp. 1-8.

17. Hassanalian, M., and A. Abdelkefi. Classifications, applications, and design challenges of drones: A review. Progress in Aerospace Sciences, Vol. 91, 2017, pp. 99-131.

18. Valavanis, K. P., and G. J. Vachtsevanos. Handbook of unmanned aerial vehicles. New York: Springer Publishing Company, Inc., 2014.

19. Zhu, X., Z. Liu, and J. Yang. Model of collaborative UAV swarm toward coordination and control mechanisms study. Procedia Computer Science, Vol 51, 2015, pp. 493-502.

20. Lee, P., S. Carman, C. K. Chan, M. Flannery, and M. Folkman. Hyperion: A 0.4 micrometer-2.5 micrometer hyperspectral imager for the NASA Earth Observing-1 mission. Redondo Beach, CA: TRW Space and Electronics Group, 2001.

21. Folkman, M. A., J. Pearlman, L. Liao, and P. J. Jarecke. EO-1/Hyperion hyperspectral imager design, development, characterization, and calibration. In Proceedings of the SPIE, Bellingham, Washington, 2001.

22. Barry, P. EO-1/Hyperion science data user's guide: Level 1B. Redondo Beach, CA: TRW Space, Defense \& Information Systems, 2001.

23. Coulter, D., P. L. Hauff, and W. L. Kerby. Airborne Hyperspectral Remote Sensing. In Proceedings of the 5th Decennial International Conference on Mineral Exploration, Toronto, Canada, 2007.

24. Green, R. O., M. L. Eastwood, C. M. Sarture, T. G. Chrien, M. Aronsson, B. J. Chippendale, and J. A. Faust. Imaging Spectroscopy and the Airborne Visible/Infrared Imaging Spectrometer (AVIRIS). Remote Sensing of Environment, Vol. 65, No. 3, 1998, pp. 227-248.

25. Kruse, F. A. Comparison of AVIRIS and Hyperion for hyperspectral mineral mapping. In The 11th JPL Airborne Geoscience Workshop, Pasadena, California, 2002.

26. Hill, S. L., and P. Clemens. Miniaturization of high spectral spatial resolution hyperspectral imagers on unmanned aerial systems. In Proc. SPIE 9482, Next-Generation Spectroscopic Technologies VIII, Baltimore, Maryland, 2015.

27. Aerialtronics International, Altura Zenith ATX8 System Overview. Katwijk, The Netherlands: Aerialtronics International, 2017. 


\section{LIST OF TABLES}

TABLE 1 Parameters of the Performance Evaluation Model

TABLE 2 Parameters of the Performance Evaluation Model

LIST OF FIGURES

FIGURE 1 Performance trade-off for hyperspectral imaging platforms.

FIGURE 2 Pushbroom method of image acquisition.

FIGURE 3 Timing of a Hyperion data collection event.

FIGURE 4 Bounds in the coverage-resolution trade-off for existing platforms. 


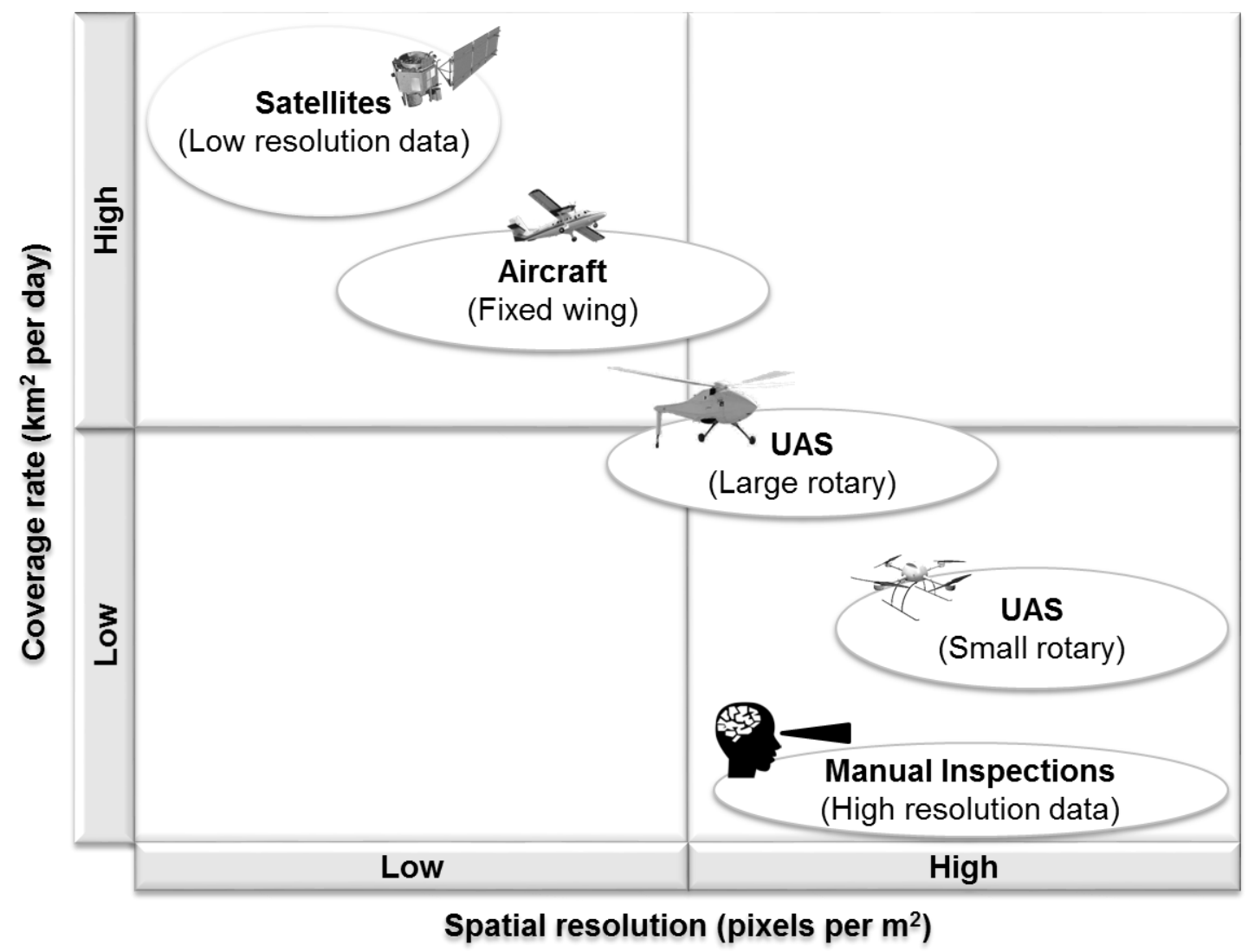

FIGURE 1 Performance trade-off for hyperspectral imaging platforms. 


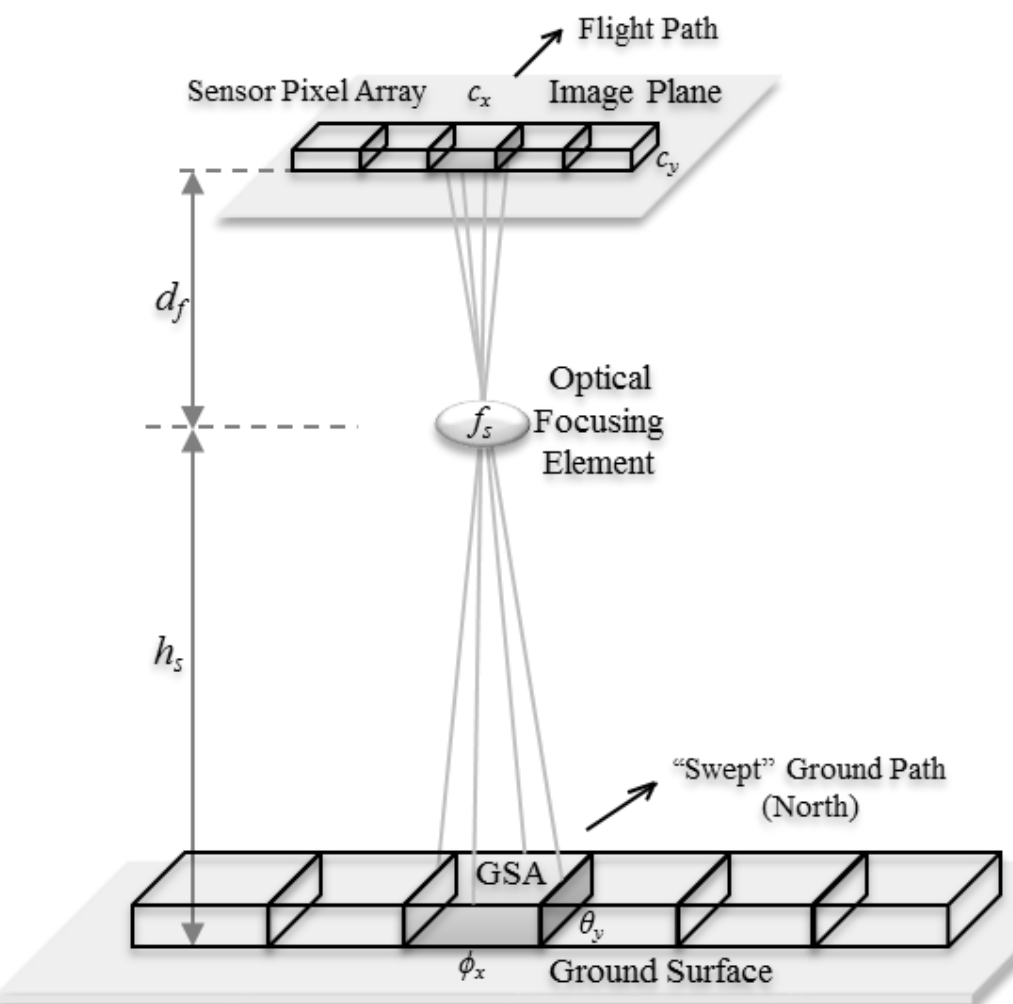

GSA $=$ Ground Sampling Area; $\phi_{x}=\phi_{y}=$ Ground Sampling Distance

FIGURE 2 Pushbroom method of image acquisition. 


\begin{tabular}{|c|c|c|c|c|c|c|c|c|c|c|c|c|}
\hline 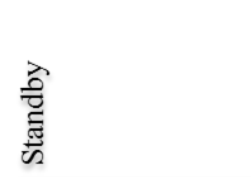 &  & 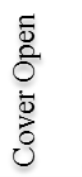 & 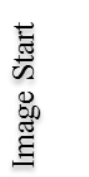 & Image Collect &  & 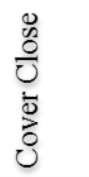 & 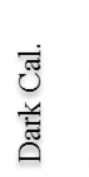 & $\begin{array}{l}\text { ठิ } \\
\text { है } \\
\text { ज్ }\end{array}$ & Lamp Cal & Lamp Off &  & $\stackrel{\underline{\underline{z}}}{\underline{\underline{z}}}$ \\
\hline$-10: 31$ & $-0: 31$ & \begin{tabular}{|l|}
$-0: 28$ \\
\end{tabular} & $-0: 03$ & 0:00 & $+0: 03$ & $+0: 11$ & $+0: 29$ & $+0: 32$ & $+3: 32$ & $+3: 37$ & $+3: 57$ & $7+4: 00$ \\
\hline
\end{tabular}

FIGURE 3 Timing of a Hyperion data collection event. 


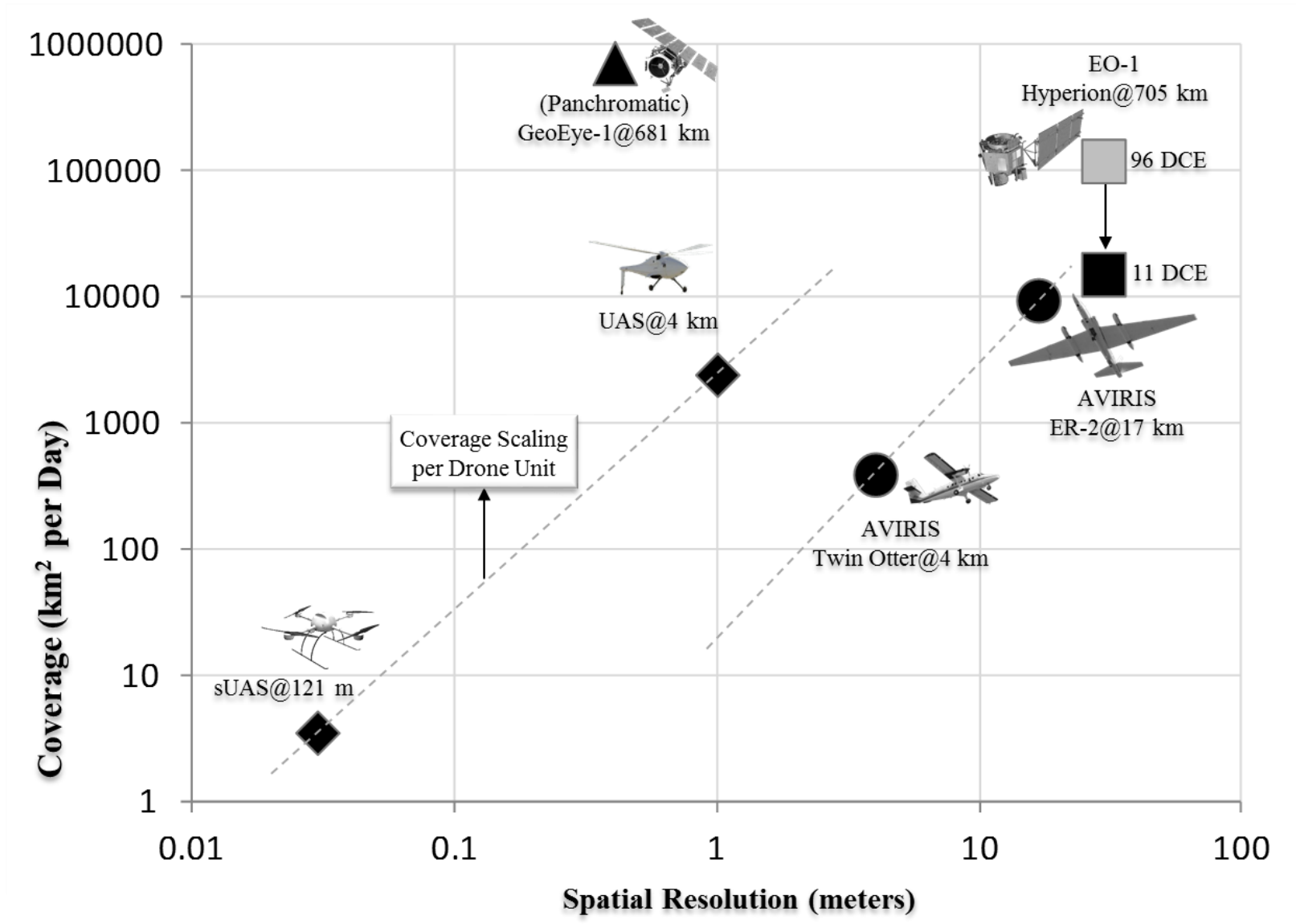

FIGURE 4 Bounds in the coverage-resolution trade-off for existing platforms. 
TABLE 1 Parameters of the Performance Evaluation Model

\begin{tabular}{|c|c|c|}
\hline Parameter & Units & Description \\
\hline$\theta$ & degrees & Latitude \\
\hline$\phi$ & degrees & Longitude \\
\hline$h$ & meters & Elevation \\
\hline$S_{\theta \phi h}$ & unit-less & Point in 3D space \\
\hline$t$ & seconds & Energy integration sample interval \\
\hline$\lambda$ & meters & Wavelength span \\
\hline$R_{\lambda}$ & Joules & Electromagnetic energy \\
\hline $\boldsymbol{p}_{x, y, i}$ & unit-less & Hyper-pixel identification number \\
\hline$G_{x, y}$ & unit-less & Center of ground sample area corresponding to hyper-pixel \\
\hline$\phi_{\mathrm{x}}$ & meters & lateral dimension of the ground sample area \\
\hline$\theta_{\mathrm{y}}$ & meters & vertical dimension of the ground sample area \\
\hline$\Delta_{\mathrm{t}}$ & seconds & Energy integration interval \\
\hline$R_{\lambda i}$ & Coulombs & Spectral charge integration vector of each hyper-pixel \\
\hline$d_{\mathrm{f}}$ & meters & Focal length of the optical system \\
\hline$d_{\mathrm{s}}$ & meters & Distance of focusing element above the image plane \\
\hline$\delta$ & meters & Separation between the image plane and the focal plane \\
\hline$h_{\mathrm{s}}$ & meters & Distance of focused subject in the ground sample area \\
\hline$c_{\mathrm{X}}$ & meters & Lateral dimension of the image sensor pixel \\
\hline$c_{\mathrm{y}}$ & meters & Vertical dimension of the image sensor pixel \\
\hline$p_{\mathrm{a}}$ & sq-meters & Pixel area \\
\hline$G_{\mathrm{A}}$ & sq-meters & Ground sensing area (GSA) \\
\hline$M_{\eta}$ & unit-less & Optical magnification factor \\
\hline$S_{\mathrm{x}}$ & meters & Image sensor length \\
\hline$N_{\mathrm{x}}$ & unit-less & Number of pixels per row of the $2 \mathrm{D}$ planar sensor \\
\hline$\gamma$ & unit-less & Image overlap fraction \\
\hline$L_{\mathrm{y}}$ & meters & Sample interval along the ground track \\
\hline$L_{\mathrm{G}}$ & meters & Length of ground (nadir) path \\
\hline$V_{\mathrm{G}}$ & $\mathrm{m} \mathrm{s}^{-1}$ & Ground speed \\
\hline$T_{\mathrm{f}}$ & seconds & Flying time \\
\hline$N_{\mathrm{F}}$ & unit-less & Number of frames needed to cover the flight path \\
\hline$F_{\mathrm{r}}$ & frames $\mathrm{s}^{-1}$ & Frame rate \\
\hline$F_{\text {rm }}$ & frames s ${ }^{-1}$ & Minimum required frame rate \\
\hline$G_{\mathrm{y}}$ & meters & Swath height \\
\hline$A_{\mathrm{n}}$ & unit-less & Number of aircrafts in the swarm \\
\hline$I_{\mathrm{V}}$ & degrees & Instantaneous field-of-view \\
\hline$W_{\mathrm{a}}$ & sq-meters & Ground area coverage \\
\hline
\end{tabular}


TABLE 2 Parameter Values of the Performance Evaluation Model

\begin{tabular}{lrrrrrrrrrr}
\hline Platform & $\begin{array}{c}h_{\mathrm{s}} \\
(\mathrm{km})\end{array}$ & $N_{\mathrm{x}}$ & $\begin{array}{c}c_{\mathrm{x}} \\
(\mu \mathrm{m})\end{array}$ & $\begin{array}{c}\phi_{\mathrm{x}} \\
(\mathrm{m})\end{array}$ & $\begin{array}{c}I_{\mathrm{V}} \\
(\mathrm{mr})\end{array}$ & $\begin{array}{c}F_{\mathrm{r}} \\
\left(\mathrm{s}^{-1}\right)\end{array}$ & $\begin{array}{c}\gamma \\
(\%)\end{array}$ & $\begin{array}{c}V_{\mathrm{G}} \\
\left(\mathrm{km} \mathrm{h}^{-1}\right)\end{array}$ & $\begin{array}{c}\eta_{\mathrm{I}} \\
(\%)\end{array}$ & $\begin{array}{r}W_{\mathrm{a}} \\
\left(\mathrm{km}^{2}\right)\end{array}$ \\
\hline Hyperion & 705 & 256 & 60 & 30 & 0.043 & 220 & 0.6 & 23,760 & 2.7 & 14,600 \\
AVIRIS-ER2 & 17 & 677 & 200 & 17 & 1.0 & 12 & 1.5 & 730 & 16.9 & 9292 \\
AVIRIS-TO & 4 & 677 & 200 & 4 & 1.0 & 12 & 25 & 130 & 16.9 & 387 \\
UAS & 4 & 1600 & 6.5 & 1 & 0.3 & 250 & 30 & 630 & 58.7 & 2,417 \\
sUAS & 0.12 & 1600 & 6.5 & 0.03 & 0.3 & 250 & 30 & 19 & 94.8 & 3.5 \\
\hline
\end{tabular}

